\title{
TTR
}

Traduction, terminologie, re?daction

\section{Interprétation et fétiches : entre traduction et psychanalyse}

\section{Gabriel Louis Moyal}

Volume 11, numéro 2, 2e semestre 1998

Psychanalyse et traduction : voies de traverse

Psychoanalysis and Translation: Passages Between and Beyond

URI : https://id.erudit.org/iderudit/037338ar

DOI : https://doi.org/10.7202/037338ar

Aller au sommaire du numéro

\section{Éditeur(s)}

Association canadienne de traductologie

ISSN

0835-8443 (imprimé)

1708-2188 (numérique)

Découvrir la revue

Citer cet article

Moyal, G. L. (1998). Interprétation et fétiches : entre traduction et psychanalyse. TTR, 11(2), 131-151. https://doi.org/10.7202/037338ar

\section{Résumé de l'article}

Interprétation et fétiches : entre traduction et psychanalyse - Si, comme le démontre George Steiner, la traduction n'est finalement qu'un mode d'interprétation, cela soulève immanquablement la question des critères pertinents et éventuellement applicables à l'évaluation de traductions. Pourtant, ce que l'on constate le mieux avec Steiner lorsqu'il évalue des traductions, c'est toute la complexité des relations entre traducteurs-interprètes et les textes qu'ils traduisent — à la fois au sens qu'elles/ils leur attribuent comme à leurs modes de le signifier. De même, l'évaluation, les jugements que l'on porte sur les traductions en disent long sur celle/celui qui évalue, sur l'importance qu'elle/il attache au sens, voire à la possibilité même de connaître ou de comprendre le monde où nous évoluons.

Tels qu'il les décrit, les premiers cas de fétichisme chez Freud opèrent de façon analogue : ils s'avèrent n'être qu'une série de tentatives d'adaptation à la réalité. Les fétiches servent à la fois à commémorer et à nier une réalité perçue mais inacceptable. Ce genre de paradoxe et les simulacres qui servent à déguiser cette réalité ne sont finalement que le tribut qu'il faut payer en échange de ce sentiment rassurant que le monde est disponible à notre entendement, que les moyens très limités dont nous disposons pour le comprendre sont adéquats. Sans critères absolus pour les évaluer, les traductions fonctionnent comme des fétiches : elles prouvent - mais provisoirement - que nous sommes capables de comprendre et d'interpréter le monde, alors que, par leur imperfection, elles réaffirment le fait que telle compréhension n'est finalement que fragmentaire.
Tous droits réservés @ C TTR: traduction, terminologie, rédaction — Les auteurs, 1998
Ce document est protégé par la loi sur le droit d'auteur. L'utilisation des services d'Érudit (y compris la reproduction) est assujettie à sa politique d'utilisation que vous pouvez consulter en ligne.

https://apropos.erudit.org/fr/usagers/politique-dutilisation/ 


\section{Interprétation et fétiches : entre traduction et psychanalyse}

\section{Gabriel Louis Moyal}

Le Chien qui lache sa proie pour l'ombre

Chacun se trompe ici-bas.

On voit couric après l'ombre Tant de fous, qu'on n'en sait pas

La plupart du temps le nombre.

Au chien dont parle Ésope il faut les renvoyer. Ce chien, voyant sa proie en l'eau représentée, La quitta pour l'image, et pensa se noyer; La rivière devint tout d'un coup agitée.

A toute peine il regagna les bords, Et n'eut ni l'ombre ni le corps. I

I

À l'école primaire que je fréquentais, il existait un tarif fixe, plus ou moins officiel, associant les peines aux délits : chaque faute avait son prix. Ne pas savoir sa leçon, ne pas pouvoir réciter le résumé d'histoire, les vers ou la table de multiplication du jour lors d'une interrogation valait au contrevenant quatre coups de règle sur chaque main. Une année, une blague idiote fit brièvement la joie des élèves de quatrième. Dans la blague, le Toto interrogé chantonnait l'air - le même que celui sur lequel

' Jean de La Fontaine, * Le Chien qui låche sa proie pour l'ombre ", fable XVII, dans Fables, Livre VI, Paris, Gallimard, * Bibliothèque de la Pléiade ", [1991], p. 232. 
nous récitions nous-mềmes indifféremment (la rime riche aidant) toutes les fables de La Fontaine (tah dah dah dah, etc.). Toto expliquait au maître ahuri - histoire de plaider pour une réduction de peine - qu'il avait pu retenir l'air, mais que les paroles lui échappaient. Nous en étions tous d'ailleurs plus ou moins au même point. Colonisés, citadins, pour qui le français était encore une langue presque étrangère, les loups, agneaux, cigales et renards parlants avaient selon nous toute la vraisemblance d'un conte de fée et moins encore de pertinence. Les illustrations des manuels d'école qui représentaient ces animaux vêtus de vestes, culottes et bonnets ne faisaient qu'en accentuer l'excentricité. Ces bêtes n'étaient pas de notre monde, elles ne pouvaient rien nous apprendre et la blague qui réduisait les fables à un air, à un chantonnement avec rime mais sans raison trouvait facilement faveur.

Pour autant, celui qui d'entre nous le premier s'avisa d'imiter le Toto de l'histoire apprit à ses dépens que notre man̂tre lui aussi connaissait la blague - et la musique. Le maître lui annonça aussi qu'au lieu d'un tarif réduit, le nouveau Toto bénéficierait d'un supplément qui lui fut administré séance tenante. Ainsi, devant nous une morale de fable se mettait en scène et prenait corps : le malin rencontrait son malin et demi, la loi du plus fort s'avérait la meilleure, etc. La fable réduite en bourrage de crâne par l'école, et jugée insipide par la gent écolière, s'imposait quand même comme vérité ou tout au moins comme leçon.

Le cancre - et nous tous qui l'avions collectivement et subtilement incité à cette bravade - avions présumé que le maître devait penser comme nous, que la fable, à force d'être ânonnée finissait par ne parler ni à nous ni à personne. Animaux pestiférés ou non, toute la ménagerie des fables et leur morale explicite n'étaient pour nous qu'autant de mots à apprendre par cour, quitte à les oublier le plus tôt possible après avoir été interrogé. C'était mal parier. L'ennui que suscitait en nous ces fables, leur langue et leur morale pour nous désuètes, ne répondaient en rien à l'attente du maître ni, derrière lui, à celle des fonctionnaires du ministère de l'Instruction publique qui les avaient mises au programme. Ce qui au mieux résonnait en nous comme autant de comptines était pour eux autant de parcelles de sagesse qu'ils avaient le devoir sacré de nous inculquer. Et là une autre morale, une autre leçon se profilait. Ce dont nous étions massivement convaincus, ce qui pour nous allait sans dire et devait immanquablement être reconnu de tous s'avérait irrecevable et 
donnait lieu à de sévères sanctions. Soudain, tout était à réviser.

Nous n'étions pas faits pour être traducteurs - et encore moins interprètes. C'est ce que George Steiner aurait ajouté s'il avait été notre maître. Pour George Steiner dans $A f t e r$ Babel, ce qui rend possible la traduction est un acte généreux : un acte de foi. C'est la foi qui fait courir le risque, qui incite à fournir l'effort, à s'investir dans l'acte de traduire. Cette foi en la signification du texte d'abord muet et indéchiffrable des autres, Steiner l'identifie comme le point de départ de tout acte de traduction :

All understanding, and the demonstrative statement of understanding which is translation, starts with an act of trust. This confiding will, ordinarily, be instantaneous and unexamined, but it has a complex base. It is an operative comvention which derives from a sequence of phenomenological assumptions about the coherence of the world, about the presence of meaning in very different, perhaps formally antithetical semantic systems, about the validity of analogy and parallel. The radical generosity of the translator ("I grant beforehand that there must be something there"), his trust in the "other "as yet untried, unmapped alternity of statement. concentrates to a philosophically dramatic degree the human bias towards seeing the wortd as symbolic, as constituted of relations in which * this " can stand for "that * and must in fact be able to do so if there are to be meanings and structures. ${ }^{2}$

Mais cet acte se révèle inévitable sans cesser pour autant d'être généreux. Chez Steiner, la traduction n'est elle-même que symptôme, échantillon d'un monde rendu nécessairement disponible à l'entendement. Sauf qu'il ne s'agit pas là tant du monde en soi ou d'un sens qui y serait ou non immanent que d'une tendance humaine. Une propension, pour ainsi dire : cette tendance à toujours vouloir retrouver le sens dans le monde. Le sens ne tient pas tant à quelque vérité qui colle à la réalité qu'à la mise en évidence d'une série de correspondances : " ceci » doit pouvoir quelque part représenter * cela ». Ce en quoi la foi, la générosité accordées aux traducteurs ne sont elles-mêmes que métonymies, témoins d'abord de cette tendance que Steiner estime inhérente à la nature humaine et qu'il infère

${ }^{2}$ George Steiner, Afer Babel: Aspects of Language and Translation, Oxford, Oxford University Press, 1992 [1975], p. 312. 
à partir de l'existence des traductions, et même du simple fait que la traduction existe. Traduire tel texte ou discours n'est d'abord que le gage rassurant et toujours renouvelable de ce que le monde peut être traduit en sens.

Et rassurer n'est pas peu de choses. Tout se trame ici comme s'il y avait, par rapport au monde, une sorte d'angoisse quant à la possibilité de l'appréhender, un besoin inné, vital et de tous les instants d'interpréter ce qui nous entoure. La traduction selon Steiner ne fait que mettre en scène à la fois l'urgence et l'inévitabilité de comprendre. Cet " élancement ${ }^{3}$ "vers le sens - notion qu'il reprend à Heidegger- en revient à nous définir : " "we are what we understand to be "entails that our own being is modified by each occurrence of comprehensive appropriation $^{+}$". Et ce geste d'appropriation révèle chaque fois une double signification. La traduction se voit plus d'une fois assimilée dans After Babel au mot anglais rapture : à la fois enchantement ou extase, mais d'où Steiner s'empresse de faire ressortir le mot * rapt ", à prendre aussi au sens français d'enlèvement. Et ce dédoublement de l'effet de la traduction par la surcharge d'un terme de même origine emprunté à une autre langue (le français, encore) se retrouve aussi dans la définition qu'initialement Steiner proposait de donner à la traduction envisagée d'abord comme interprétation. Là encore l'anglais est à court de ressources :

Through the engagement of his own identity, a critic becomes un interprète - a life-giving performer - of Montaigne or Mallarme. As it does not include the world of the actor, and includes that of the musician only by analogy, the English term interpreter is less strong.

${ }^{3}$ Ibid, p. 319.

"Ibid., p. 315. Voir aussi, ibid., pp. 313-314: "The postulate that all cognition is agressive, that every proposition is an inroad on the world, is, of course. Hegelian. $I t$ is Heidegger's contribution to have shown that understanding. recognition, interpretation are a compacted, unavoldable made of attack. We can modulate Heidegger's insistence that understanding is not a matter of method but of primary being, that 'being consists in the understanding of other being' into the more naive, limited axiom that each act of comprehension must appropriate another entity (we translate into)." 
But it is congruent with French when reaching out in another crucial direction. Interprète/interpreter are commonly used to mean translator.'

Surtout l'intention expresse est de reconsidérer la traduction moins comme une visée vers l'état statique du texte qu'une dynamique vitale, soutenue, toujours prégnante. En cela, ces recours aux couches interlinguistiques du sens des mots, ces appels à des connotations enfouies dans une étymologie commune à plusieurs langues miment le propos steinerien. Les textes une fois traduits tendent à pétrifier le sens des mots; l'interprétation comme dynamique constante en connote toujours plusieurs à la fois : elle mélange les codes, les juxtapose, les brouille. La langue cible de l'interprétation se doit d'être mouvante. L'interprète selon Steiner s'évertue à en fouiller inlassablement les couches parlantes.

Mais en réinscrivant la traduction dans l'entreprise plus vaste de l'interprétation, en l'envisageant comme procès plutôt que comme produit, c'est surtout l'engagement de l'interprète que Stejner met en cause. Dans les analyses de versions qu'il présente, il ne s'agit pas tant d'illustrer la compétence linguistique des traducteurs que de mettre en lumière la perspective philosophique et culturelle de l'interprète - lequel est aussi souvent auteur, poète, penseur, etc. En outre, si l'original et la version invitent à comparer les harmonies et discordances des codes linguistiques et culturels impliqués, pour Steiner, ils confrontent aussi à des personnalités d'autant plus exposées qu'elles sont exacerbées par la nécessité de négocier entre les limites et exigences redoublées de ces codes - de leurs conventions et de leurs contraintes. C'est ce qu'on peut constater, par exemple, dans cette analyse de la traduction des Fables de La Fontaine par la poétesse américaine Marianne Moore :

In Marianne Moore's La Fontaine (1954), the control of temporal distances is complex and brilliant. Though Miss Moore professes herself to be entirely Pound's disciple - the natural order of words, subject, predicate, object; the active voice where possible; $a$ ban on dead words, rhymes synonymous with gusto "- her practice is in fact highly idiosyncratic. It comprises extreme verbal decorum, often

${ }^{5}$ Ibid., p. 28. $\dot{A}$ la ligne, Steiner ajoute cette seule phrase : "This, I believe, is the vital starting point." 
precisely attuned to the speech-habits of women in nineteenth-century New England, an arch pleasure in the Latinate and technical terms, tricks of compaction which owe something to the elided grammar of Emily Dickinson, and a pattern of enjambment and prim caesura which are the mark of her own verse. The cunning plainness of the Fables, La Fontaine's admixture of colloquial with neo-classical modes, exactly suited Marianne Moore's gifts."

L'auteure Moore a beau prétendre, chercher à se dissimuler sous les traits stylistiques de ceux dont elle se veut l'émule, pour peu qu'elle s'adonne à la traduction, dès qu'elle interprète et/ou performe les Fables, sa vraie nature - les forces, les faiblesses de son écriture - se dévoilent. Et ce sont les secrets jusque-là les mieux occultés de son style qu'elle se trouve avoir inconsciemment mis à nu. Mais il n'y a pas bien entendu que la nature poétique de Marianne Moore qui soit révélée dans et par sa traduction. Quelque chose de La Fontaine, quelque " admixture of colloquial with neo-classical modes " se voit pareillement mis en lumière, accentué dans la rencontre avec Moore. La traduction - comme toute forme d'interprétation - comporte inévitablement cet effet de réciprocité. Et cela vaut aussi pour les interprétations, analyses, évaluations, etc. de traductions, comme celles, par exemple, que pratique Steiner; et pas moins, naturellement, celles que l'on peut lire dans le présent essai.

Mais la traduction semble surtout donner à Steiner l'accès à quelque vérité plus profonde sur l'auteur qui traduit ainsi que le texte et l'auteur qui sont traduits. Comme si, partagés entre deux langues, ils devenaient chacun plus transparents. Comme si, en s'évertuant à vêtir les mots d'un autre code que ceux du sien propre, à vouloir en cemer l'équivalent le meilleur, on en laissait d'autant mieux parâtre la forme, on rendait paradoxalement plus visibles les traces de ces lignes qui démarquent l'idée de l'expression, l'essentiel du contingent. C'est là où on les croirait le mieux camouflés, tendus entre deux codes, jouant dans le registre d'un autre et dans le leur à la fois, que pour Steiner ces auteursinterprètes deviennent le plus * eux-mêmes $»$. Il y a là comme un effet $a$ contrario : plus les différences sont accusées plus l'identité transparaît.

${ }^{6} \mathrm{lbid}$, p. 368. Ce qui accentue ce sentiment que l'attention porte d'abord sur la personnalité des auteurs et interprètes est sans doute cette présence incontournable de Steiner, de sa culture encyclopédique, de sa perspicacité stylistique, etc. 
Et dans tout cela, Steiner qui, pour sa part, semble ne jamais pouvoir se dissoudre, se perdre lui-même de vue au sein de ce Babel, de la multitude de langues qu'il maîtrise, ne perd pas de vue non plus l'unité autour de laquelle les auteurs-traducteurs qu'il analyse finissent par graviter. Il y a à cela la même urgence, la même angoisse qui s'obstine à comprendre, à retrouver un sens dans le monde. Mais si l'interprétation chez Steiner reste en mouvement perpétuel, ici, dans cette tension où sont pris les poètes traducteurs, la dynamique du sens semble s'amortir : plutôt qu'une multiplicité de sens en flux, une vérité, une version unique commence à émerger, à se mettre en place. L'art de la poétesse, celui du fabuliste commencent respectivement à se profiler dans leur essence profonde. Au lieu de se fondre l'une dans l'autre successivement, les images tendent à se cristalliser. Si nous sommes ce que nous comprenons être (" we are what we understand to be ${ }^{7}$ ), identifier, discemer les modes d'être des autres conforte le sens que nous avons d'être, nous renvoie l'image raffermie de nous-mêmes.

\section{III}

Cet effet de renvoi d'image n'est pas exclusif, tant s'en faut, à la traduction de textes, de thèmes et de versions écrites. La psychanalyse qui, dans une large mesure, informe cette lecture du texte de Steiner est d'autant plus sujette a ces effets de réciprocité entre ce qui est à première vue interprété et celle/celui qui pense interpréter qu'elle s'est donné d'emblée, comme règle de procédure, l'implication personnelle de l'analyste dans l'interprétation. En outre, les interventions de la traduction dans la pratique psychanalytique ne se limitent pas à l'interprétation savante attendue de l'analyste. Dans la mesure où la psychanalyse dépend avant tout d'un rapport vécu entre personnes, elle est à considérer comme une pratique dynamique. La relation de cas, la transcription du processus analytique pourraient donc être compris comme entretenant des rapports analogues à ceux entre les textes traduits et l'interprétation comme «performance ".

Mais cette sorte d'analogie tend à trop simplifier. La théorie de la traduction, les analyses critiques de traductions existantes qu'opère

\footnotetext{
${ }^{7}$ Ibid., p. 315.
} 
Steiner ne sont pas tout uniment analogues à la théorisation en psychanalyse, à quelque métapsychologie détachable de la pratique analytique en soi. Tout comme les analyses de traductions chez Steiner sont elles-mêmes des instances d'interprétation, les interprétations de symptômes, de discours, etc. en psychanalyse sont aussi des analyses de modes plus ou moins adéquats d'adaptation, de compréhension ou d'intégration au monde tentés par les patients. Ce que l'analyste finit par interpréter, ce sont aussi souvent des interprétations plus ou moins clairement esquissées du monde.

Deux études de Freud sur le fétichisme mettent succinctement en scène cette interaction, ce jeu entre les interprétations. Dans le premier état disponible de ses travaux sur le fétichisme, Freud voulait distinguer parmi les instances de fétichisme répertoriées par Kraftt-Ebing (et d'autres), entre celles qu'on peut saisir et différencier clairement et celles qui restent énigmatiques. Il proposait de retenir le nom de fétiche pour ces dernières. Les autres, il fallait les appeler par leur nom, par quoi il entendait vraisemblablement les reclasser selon des critères psychanalytiques'. Les cas énigmatiques devenaient ainsi ceux où le fétiche n'avait rien à voir avec l'amour, n'était pas constitué par les attraits

${ }^{3}$ Louis Rose, édit. et trad., = Freud and Fetishism: Previously Unpublished Minutes of the Vienna Psychoanalytic Society „, Psychoanalytic Quarterly, LVII, 1988 [dorénavant représenté dans ces notes par l'abréviation * édit. Rose "]. II s'agit des minutes d'une présentation de Freud à la société de Vienne le 24 février 1909 sur * La genèse du fétichisme *. Ces notes ont longtemps été considérées perdues jusqu'à ce que Louis Rose les ait retrouvées dans l'archive Rank à l'Université Columbia à New York. L'édition francaise des minutes (Les Premiers Psychanalystes: Minutes de la Societe psychanalytique de Vienne, 4 vol., Paris, Gallimard, 1976-1983) n'a donc pu que faire état de leur disparition, (voir op. cit., vol. II, p. [163]). Le trajet qu'elles ont pu suivre est esquissé dans l'introduction à la traduction de Louis Rose.

${ }^{9}$ Voir Rose, p. 153 : "Many obscure points inhere in this subject. Above all, in Ebing as much so as in others, diverse phenomena are understood by the concept of fetishism. 1) Things which we can grasp and distinguish exactly, and 2) something puzzling; it is suggested that the name 'fetish' be reserved for the latter, the puzzling, and that the others be called by their appropriate names." 
sexuels " normaux " (les seins, les cheveux, etc. $)^{10}$. Ainsi en venait-il au cas d'un homme dont le fétiche était le vêtement féminin - ce en quoi il était très exigeant, la moindre négligence vestimentaire chez la femme qu'il fréquentait entrainant une rupture de relations". Mais avant de devenir fétichiste, ce patient, par ailleurs impuissant, était aussi, par une longue intimité avec sa mère, devenu voyeur. Et c'est pour réprimer le plaisir coupable de la contemplation de la nudité maternelle, explique Freud, qu'il était devenu fétichiste : " he becomes a clothes fetishist out of repression of the desire to look ${ }^{12}$.

Mais avant d'en arriver là, Freud avait noté, comme en passant, une autre forme qu'avait prise le fétichisme de ce patient :

Some parallels to his interest in clothes occur in other of his characteristics. The patient became a speculative philosopher, and names play for him an especially great role. In this patient something similar to what took place in the erotic domain occurred in the intellectual domain : he turned his interest away from things onto words, which are, so to speak, the clothes of ideas; this accounts for his interest in philosophy. ${ }^{13}$

Comme par analogie avec sa passion pour le vêtement - ou la nudité le patient était devenu un " philosophe spéculatif ", un habilleur/déshabilleur d'idées, laisse entendre Freud ${ }^{14}$.

${ }^{10}$ Ibid., pp. 152-153.

"Ibid., p. 154.

${ }^{12}$ Ibid., p. 155, souligné dans le texte.

${ }^{13}$ Ibid., p. 154.

${ }^{14}$ Il ne faudrait pas perdre de vue ici qu'il ne s'agit pas là d'un texte de Freud, traduit ou original, mais de minutes, d'une transcription - vraisemblablement par Rank - d'une présentation faite par Freud en 1909, soit quelque dix-huit ans avant la publication de son article sur le fétichisme. 
Ce qui frappe surtout dans le cas de ce patient c'est ce qu'on pourrait appeler la fluidité des traductions qui ont cours en lui. Le patient passe du voyeurisme au fétichisme (sous deux formes : il a aussi une prédilection marquée pour les pieds et leur odeur), et de là à la philosophie - et, sans doute, ailleurs ensuite - comme si c'était quelque part " la même chose ". Sauf que pour que toutes ces choses très différentes (voire contradictoires) s'avèrent n'être que les diverses versions d'une seule, il faut passer par l'analyste à qui le patient les confie. Le patient, pour sa part, reste un virtuose du déguisement : il a trouvé le moyen de rendre indépistable(s) son (ses) fétiche(s) tout en en multipliant infiniment la jouissance. Qui d'autre que Freud pourrait jamais comprendre qu'en décortiquant inlassablement les mots il revisitait impunément la nudité de sa mère ou se laissait encore aller à rajuster le col de quelque maîtresse débraillée ${ }^{15}$ ?

On pourrait même se demander si ce patient ne finit pas par déjouer la perspicacité de Freud. Car en même temps qu'il s'adonne à la transposition infinie de ses secrètes jouissances, Freud, lui, chercherait, à partir de ce cas, à renfermer dans une seule et même explication toutes les manifestations du phénomène à venir, tous les cas de fétichisme. C'est du moins ce qui transparaît dans les minutes de cette présentation de cas où Freud fait état à la fois des faiblesses et néanmoins de l'importance fondamentale de ses conclusions :

The weak side of this attempt is that it rests entirely on the observation of three instances divided among two persons. On the other side.

${ }^{15}$ Dans * Le fétichisme * (dans La vie sexuelle, Paris, PUF, 1969 [1927]), Freud discute brièvement d'un fétiche dont le secret est gardé par un effet de traduction : - Le cas le plus remarquable était celui d'un jeune homme qui avait érigé comme condition de fétiche un certain "brillant sur le nez". L'explication surprenante en était le fait qu'élevé dans une nurserie anglaise, ce malade était ensuite venu en Allemagne où il avait presque totalement oublié sa langue matemelle. Le fétiche dont l'origine se trouvait dans la prime enfance ne devait pas être compris en allemand mais en anglais; le "brillant sur le nez" était en fait un "regard sur le nez" [Glanz/glance] ; ainsi le nez était ce fétiche auquel, du reste, il pouvait à son gré octroyer ce brillant que les autres ne pouvaient percevoir - (Freud, op. cit., p. [133]). 
however, this explanation is of so fundamental a nature, one can assume that other cases will behave similarly. ${ }^{16}$

Rien dans les minutes en dehors de cette affirmation attribuée à Freud n'indique ce qui aurait pu justifier cette anticipation que les conclusions auxquelles il en vient ici pourraient s'appliquer ou servir à expliquer d'autres cas. Devant l'étendue et la diversité des métamorphoses du fétichisme de ce patient on ne peut que très provisoirement expliquer cette anticipation par un enthousiasme nourri par le démon de l'analogie, le jeu de la métaphore qui se file et laisse se découvrir une trame, une constante centrée sur le regard et sur la différence entre ce qu'en fait il y aurait à voir et ce qui serait perçu ${ }^{17}$. Loin de se laisser dérouter, la confiance de Freud en la cohérence ultime de ces symptômes et de ces comportements s'affine à mesure que le fétichiste transpose et prodigue de nouvelles versions de sa passion ${ }^{18}$. Ici aussi, comme chez Steiner, la présence de l'interprète se fait particulièrement sentir : dans cet enthousiasme qui pousse Freud à avancer une théorie unique et de portée universelle devant cette éclosion de symptômes, devant la légion de formes à travers

${ }^{16}$ Rose, p. 154. Dans la version très différente de la théorie du fétichisme de 1927 , Freud affichera la même conviction, le même optimisme quant à l'applicabilité de la nouvelle théorie aux cas à venir : " Les renseignements foumis par l'analyse sur le sens et la visée du fétiche étaient les mêmes dans tous les cas. Ils se déduisaient si spontanément et m'apparurent si contraignants que je suis prêt à m'attendre à ce que tous les cas de fétichisme aient une même solution générale. *

${ }^{17}$ Le regard est le principal élément qui, de ce premier travail sur le fétichisme, sera retenu dans l'article de 1927.

${ }^{18}$ Malgré le jeu de mots très suggestif sur le regard du voyeur et la philosophie " spéculative ", aucune trace ne subsiste dans les minutes que Freud se soit attardé a donner sur ce cas d'illustrations de l'importance des mots dans la vie du patient. Cf. pourtant ce commentaire d'Ilse Grubrich-Simitis sur la place des mots dans le travail de Freud : [ [...] beaucoup de notations témoignent de la fascination de Freud pour la langue et de son génie linguistique. Elles donnent l'impression d'être les restes d'un travail de traduction permanent multidimensionnel - du symptôme au conflit inconscient, du contenu du rêve manifeste au contenu du rêve latent, de la constellation du transfert à la scène d'enfance, de la fantaisie originaire aux événements réels de la phylogenèse * (Ilse Grubrich-Simitis, Freud : Retour aux manuscrits, Paris, PUF, 1997 [1993], p. 128). 
lesquelles passe ce fétichisme très particulier. Il ne faut pas qu'il manque d'assurance pour en arriver à une seule et même explication alors qu'en même temps il admet ne pas encore avoir pu accumuler toutes les observations nécessaires ${ }^{10}$.

Dans ces premiers travaux sur le fétichisme Freud s'inscrit lui aussi dans cette tendance à interpréter par élimination des contradictions (le fétichisme remplace le voyeurisme refoulé; il $\mathrm{y}$ a les fétiches * normaux * et ... les autres), à trouver le sens dans l'accumulation et la cohérence des analogies. L'assurance affichée par Freud dans la relation de ce cas avalise l'interprétation proposée quant à son extension à d'autres cas éventuels : les différences et particularités paraissent devoir s'estomper en faveur d'une cohérence du discours qui vraisemblablement en rehausse la teneur et le prestige scientifiques. Freud semble y gagner assez de confiance pour projeter encore plus loin ses propres spéculations : il renverse, il retoume comme un gant cette théorie d'un fétichisme né du refoulement d'un autre instinct pour l'appliquer aux femmes, à l'histoire de la mode en général, au culte de la Vierge au Moyen Âge, etc. ${ }^{20}$.

${ }^{19}$ Voir aussi cette remarque préliminaire au début des minutes : "Departing from his principle not to formulate theory before it can be supported by observations, the speaker would like to communicate, by way of exception, a theory of fetishism which is based only on a small number of observations "(Rose, op. cit., p. 150; je souligne).

${ }^{20}$ Voir, ibid., pp. 155-156: ${ }^{a}$ This explanation of clothes fetishism is actually no novelty. In the world of everyday experience, we can observe that half of humanity must be classed among the clothes fetishists. All women, that is, are clothes fetishists. Dress plays a puzzling role in them. It is a question again of the repression of the same drive, this time, however, in the passive form of allowing oneself to be seen, which is represented by clothes, and on account of which. clothes are raised to a fetish. Only now we understand why even the most intelligent women behave defenselessly against the demands of fashion. For them, clothes take the place of parts of the body, and to wear the same clothes means only to be able to show what the others can show, means only that one can find in her everything that one can expect from women, an assurance which the woman can give only in this form. Otherwise it would be incomprehensible why many women, following the demands of fashion, also want to wear, and do wear, pieces of clothing which do not show them to their best advantage, which do not suit them." 
Comme ce patient fétichiste, la théorie de Freud fait montre d'une remarquable adaptabilité. Dans son agilité, dans sa capacité virtuellement infinie d'expliquer, elle devient elle aussi - du moins sur le mode de sa performance, de sa dynamique - ce qu'elle cherche à comprendre.

Mais la question se pose aussi quant au rôle de l'analyste. Devant cette théorie polymorphe qui commence à ressembler à une machine à interpréter, on peut se demander ce qu'ont pu être les modalités de l'implication de l'analyste, les circonstances et la nature du rapport au patient qui aboutissent à une théorisation en apparence si englobante. Là où Steiner jouait sur une tension entre les codes, Freud semble d'abord miser sur une certaine élasticité, sur la générativité d'une théorie capable de s'accorder aux variations de l'expérience, de produire des variantes d'elle-même sans pour autant entamer sa propre cohérence. L'enjeu, audelà de la théorie en soi, serait ici aussi - comme chez Steiner - la crédibilité du théoricien, la stabilité d'une identité unique, capable vraisemblablement de tout expliquer.

Mais il s'agit là d'une étape dans l'évolution d'une interprétation. Et qui plus est, d'un texte apocryphe, un compte rendu de présentation signé d'une autre main. Même si certains aspects de ce premier essai sur le fétichisme seront repris dans l'essai de 1927 (le dosage de cas et de théorie, les projections anticipées sur d'autres cas, les tentatives d'application dans le domaine culturel), les perspectives et les conclusions des deux textes s'avèrent fondamentalement différentes. Là où, dans la première vérsion, Freud se mettait en scène en se prévalant d'un mode d'interprétation à toute épreuve, dans la dernière il fondera son interprétation sur une théorie de la fragilité du savoir.

\section{IV}

Dans son article sur la traduction de la Standard Edition des æuvres de Freud, Bruno Bettelheim ${ }^{21}$ reprenait justement ce problème de l'investissement personnel du théoricien, de l'analyste à la fois dans sa

2l Bruno Bettelheim, "Reflections: Freud and the Soul ", The New Yorker, March 1 ${ }^{\text {st }}$, 1982, pp. 52-93 (dorénavant, * Bettelheim ", suivi de la page). 
recherche en elle-même - en tant que savoir - et dans la projection de celle-ci en modèle pour les praticiens à venir. Bettelheim s'indignait de voir combien la traduction de Strachey qui informait (et informe encore) la pratique psychanalytique (surtout mais pas uniquement dans le monde anglophone) avait détoumé les intentions de Freud. Pour Bettelheim, cette traduction avait transformé ce qui devait être une science humaine en une science naturelle. Cette déformation de Freud, Bettelheim l'attribuait au désir des traducteurs, à leur personnalité collective qui, selon lui, transparaît dans la traduction. Comme chez Steiner - mais ici, accessoirement seulement - la traduction s'avère aussi révélatrice, épreuve de vérité :

I do not doubt that Freud's English translators wanted to present his writings to their audience as accurately as possible -within the frame of reference within which they wished him to be understood. When Freud appears to be either more abstruse or more dogmalic in English transtation than in the original German. to speak about abstract concepts rather than about the reader himself, and about man's mind rather than about his soul, the probable explanation isn't mischievousness or carelessness on the translators' part but a deliberate wish to perceive Freud strictly within the framework of medicine, and, possibly, an unconscious tendency to distance themselves from the emotional impact of what Freud tried to convey. ${ }^{22}$

II faudra revenir sur cette interprétation, sur l'explication hypothétique et très typiquement psychanalytique des choix faits par Strachey. Mais il importe de noter aussi qu'il ne s'agit pas uniquement d'une critique linguistique ou stylistique de la traduction de Strachey, mais bien des effets historiques que les choix opérés - l'option systématique en faveur d'une terminologie médico-scientifique - auront entraînés, au cours de longues années, sur la pratique et le développement des institutions psychanalytiques en Amérique, à savoir, entres autres, l'évolution de la psychanalyse dans le cadre de la psychiatrie médicale aux États-Unis, l'exclusion quasi totale de l'analyse laïque, etc. Bettelheim ne peut que déplorer ces effets qu'il attribue aussi à la traduction de Strachey. Surtout, à partir de sa propre expérience, à partir de l'enseignement et de la formation qu'il a été appelé à dispenser, Bettelheim ne peut s'empêcher de

${ }^{22}$ Steiner, op. cit., p. 63. 
constater l'écart grandissant qu'il y a entre la pratique américaine et les intentions pour lui évidentes dans le texte freudien :

For nearly forty years, I have taught courses in psychoanalysis to American graduate students and residents in psychiatry. Almost invariably, I have found that psychoanalytic concepts had become for these students a way of looking only at others, from a safe distance nothing that had any bearing on them. They observed other people through the spectacles of abstraction, tried to comprehend them by means of intellectual concepts, never turning their gaze inward to the soul or their own unconscious. This was true even of the students who were in analysis themselves - it made no appreciable difference. ${ }^{23}$

En traduisant comme il l'a fait, Strachey aurait donné libre cours à cette approche très pragmatique des écoles psychiatriques américaines. Essentiellement la traduction de Strachey a autorisé sinon encouragé f'érosion de ce principe - vital, selon Bettelheim - de l'implication personnelle nécessaire de l'analyste dans l'interprétation comme dans le traitement. La réticence de Strachey devant l'investissement émotionnel qu'exigeait le texte freudien - ce a quoi Bettelheim attribue hypothétiquement les choix particuliers de terminologie $-s$ 'est donc đémultipliée et a engendré des générations d'analystes conformes à ce modèle : des analystes qui croient devoir garder envers leur pratique thérapeutique la distance émotionnelle que maintiendrait un technicien de laboratoire envers les microbes qui passent sous son microscope $\mathrm{e}^{24}$.

${ }^{23}$ Ibid., p. 52.

24 L'évolution actuelle de la psychanalyse en Amérique du Nord n'a fait que confirmer les appréhensions de Bettelheim. Le sort lié de la psychanalyse à la psychiatrie semble, en Amérique, avoir aujourd'hui abouti à la détérioration des principes psychanalytiques freudiens, dans la pratique comme dass l'enseignement. Et ces détournements de la doctrine psychanalytique n'ont pas suffi à empêcher - ou plutôt, ont sans doute aidé - à discréditer les psychothérapies interpersonnelles en faveur de traitements psychopharmaceutiques considérés comme plus efficaces. Ainsi, par exemple, dans les demières années, les médias ont trouvé utile ou facile d'associer régulièrement le nom de Freud à divers scandales provoqués par le * False Memory Syndrome ". Voir ce numéro spécial du magazine américain Time, "Is Freud Dead? " (29 novembre 1993), ou encore la série d'articles et la correspondance publiées dans la New York Review of Books (18 novembre 1993, 5 février 1994). 
Mais pour mettre en question l'évolution de la psychanalyse en Amérique et ailleurs où la traduction de Strachey a pu avoir cours et influence, Bettelheim ne se fonde pas sur sa seule connaissance de l'allemand, sur sa perception des subtilités linguistiques qui auraient échappé aux traducteurs comme aux lecteurs éventuels de cette version. Bettelheim commence par une expérience personnelle, plus intime, qu'il expose comme étant, sur un bon nombre de points déterminants quant à la portée du texte freudien, identique à celle de Freud. C'est ce qui se lit dès les premières lignes de $\mathrm{l}^{\prime}$ article :

As a child born into a middle-class, assimilated Jewish family in Vienna, I was raised and educated in an environment that was in many respects identical with the one that had formed Freud's background. The culture that was transmitted to me in my home, then in secondary school, and finally, at the University of Vienna, had changed very little since Freud's student days, fifty years earlier. So it was natural that from the time I began to think on my own I read Freud. ${ }^{25}$

Bettelheim a connu le même monde, les mêmes écoles que Freud simplement avec cinquante années de retard au cours desquelles rien n'aurait guère changé. S'il peut s'en prendre à Strachey pour avoir systématiquement remplacé l'âme (psyche) par l'intellect (mind) ou, plus généralement, pour avoir introduit une terminologie alourdie de termes techniques grecs et latins là où Freud voulait expressément s'en tenir à des mots de tous les jours, c'est parce qu'il a parlé la même langue dans, à peu de choses près, le mềne registre que Freud.

Pourtant, ce qui retient l'attention dans ce passage est la forme que prend l'expression de cette identification de la part de Bettelheim : « [...] so it was natural that from the time I began to think on my own I read Freud. w $^{26}$ L'expression " so it was natural... " introduit normalement une conclusion logique, le résultat inévitable, ou presque - " natural " de ce qui précède. Dans la logique du paragraphe (et de l'article, puisque c'en est le début), il est " natural ^ de lire Freud après avoir eu la même

${ }^{25}$ Bettelheim, art. cité, p. 52.

${ }^{26} \mathrm{Ibid}$. 
éducation, après avoir fréquenté les mêmes écoles que lui. On peut se demander ce qui dans cette communauté d'expérience rend la lecture de Freud inévitable. Surtout que l'identification se présente à la fois comme subtilement motivée par ce parcours commun et comme déclaration d'autonomie : "from the time I began to think on my own ". L'expression est difficile à traduire dans ce contexte, mais on pourrait provisoirement la rendre par : dès lors que je me mis à formuler mes propres idées $n$, ou encore : " dès que j'eus atteint l'âge de raison ". Comme si pour Bettelheim penser seul c'était - inévitablement - penser avec Freud, penser en lisant Freud.

Malgré l'ambiguité, les problèmes de lecture que ce segment de phrase peut poser, on ne saurait le considérer comme un lapsus - du moins pas dans le sens où il viendrait contredire ou infirmer les intentions explicites de l'ensemble de l'article. Au contraire, cette inférence vient renforcer l'autorité de Bettelheim, son droit de parler au nom de Freud, de mieux traduire sa parole. Peut-être Bettelheim cherche-t-il à nous faire voir - comme par le biais de cette ambiguitté même - qu'il s'était identifié à Freud au point de ne plus savoir exprimer clairement toute la mesure de cette identification. Il en était arrivé à ce point où, pour lui, penser indépendamment impliquait inévitablement la lecture de Freud. Et cette lecture, puisqu'elle se faisait en allemand et non en version anglaise, l'accaparait, dissolvait tout écart possible.

Pour dénoncer les traducteurs de la Standard Edition, pour dénoncer en particulier la distance qu'ils avaient prise et cherché a enseigner afin de se garantir de toute contamination, de tout investissement, Bettelheim se met en scène. Il expose son identification à Freud. Et il le fait sur le mode d'une inadvertance grammaticale, d'une ambiguitté d'expression qui, intentionnelle ou non, ne peut que souligner l'authenticité de cette identification.

Dans cette évaluation critique de la traduction de la Standard Edition - des raisons et des conséquences de cette traduction -, Bettelheim nous laisse lire encore plus ouvertement cet effet inévitable de l'interprétation : que ce que les interprétations tendent à révéler a au moins autant à voir avec l'interprète qu'avec ce qu'elle/il croit interpréter. L'autre de l'interprète - texte ou auteur -, l'interprète le constitue d'avance. Comme chez Steiner, cet autre ne sert le plus souvent que de prétexte à la 
révélation, à consolider cet enracinement qui ne nous est accessible que par voie de compréhension.

Chez Freud, cet effet de l'interprétation, l'implication inévitable de l'interprète, renvoie à une conception du savoir en évolution perpétuelle. Le savoir qui oriente nos pratiques n'a pas cette forme systématique et méthodique qu'on a longtemps voulu lui attribuer. La notion d'objectivité scientifique telle qu'elle s'imposait à l'époque où Freud formulait ses théries ne correspondait en rien à la conscience qu'il avait lui-même de sa propre appréhension de la réalité. L'observation, la description, l'organisation des phénomènes à étudier subissaient inévitablement, selon lui, l'influence de l'observateur ${ }^{27}$. Et cela il avait aussi à le constater régulièrement dans l'analyse de ses patients, dans cette capacité qui ne leur était pas exclusive de faire cohabiter dans leur esprit des versions contradictoires de la réalité.

Un exemple probant nous est fourni par l'article que Freud consacre au fétichisme en 1927. Dans cette dernière version Freud annule l'effet d'exclusion, de substitution d'un symptôme par le refoulement d'un autre. Le fétiche n'est plus ce reste bien enfoui, issu du refoulement d'un instinct honteux. Plutôt, le fétiche paraît la résultante d'une cohabitation

${ }^{27}$ Cest ce qu'on peut lire, par exemple dans ce passage de * Pulsions et destins des pulsions *: * Nous avons souvent entendu formuler l'exigence suivante : une science doit être construite sur des concepts fondamentaux clairs et nettement définis. En réalité. aucune science, même la plus exacte, ne commence par de telles définitions. Le véritable commencement de toute activité scientifique consiste plutốt dans la description de phénomènes, qui sont ensuite rassemblés, ordonnés et insérés dans des relations. Dans la description, déjà, on ne peut éviter d'appliquer au matériel certaines idées abstraites que l'on puise ici ou là et certainement pas dans la seule expérience actuelle. De telles idées - qui deviendront les concepts fondamentaux de la science - sont dans l'élaboration ultérieure des matériaux, encore plus indispensables. Elles comportent d'abord nécessairement un certain degré d'indétermination; il ne peut être question de cerner clairement leur contenu * (" Pulsions et destins des pulsions $*$, dans Métapsychologie, Jean Laplanche et J.-B. Pontalis édit. et trad., Paris, Gallimard, 1968 [1915], p. [11]). 
impossible : celle justement de deux versions foncièrement incompatibles de la réalité. Le fétiche sert à la fois à nier que la femme n'a pas ce pénis que le petit garçon lui avait attribué en l'imaginant formée à son image, et, en même temps, à commémorer la découverte que cette projection narcissique était erronée $e^{28}$. La révélation de l'erreur n'entraîne pas nécessairement la renonciation à l'erreur. L'enjeu de l'erreur dépasse la croyance ponctuelle et les circonstances de son discrédit. Car ce que la révélation de l'erreur met en cause pour l'enfant est d'abord la capacité qu'il a ou n'a pas de transiger avec le monde, d'y trouver sa place en l'interprétant adéquatement. La menace de castration que provoque l'absence du pénis matemel est en même temps celle de l'ineptie. La peur panique déclenchée est autant celle de ne pas avoir ce qu'il faut pour comprendre et survivre dans le monde que la menace de perdre ce qu'il a déjà.

Cette menace d'ineptie, d'insuffisance et les fétiches cognitifs qui peuvent servir à la conjurer ne sont pas exclusivement des effets pathologiques, des comportements marginaux propres aux patients. Dans la logique du savoir qui, chez Freud, procède d'erreur en étonnement, de projection narcissique en révélation, ils s'avèrent, avec le bon sens, les choses đu monde les mieux partagées. On ne peut sans doute s'empêcher de vouloir surmonter cette menace constante, de chercher ou de brandir les gages de la certitude, les marques d'une interprétation du monde sans faille ni faiblesse. On ne peut non plus renoncer à apprendre, à trouver le sens pour avoir compris que telle compréhension, telle interprétation ne pourra éviter de nous renvoyer aussi l'image de notre ineptie et de nos insuffisances. Il ne faudrait pas non plus, comme le chien de cette fable qu'on ne nous apprenait pas, chercher cet objet insaisissable du savoir qui, pour paraître ne pas avoir de lien avec nous, ne nous exposerait plus, ne renverrait plus notre image. Comme le montre La Fontaine qui, pour avoir traduit Ésope, devait en savoir quelque chose, dans cette entreprise les risques sont encore plus grands.

McMaster University

${ }^{28}$ Voir * Le fétichisme *, op. cit., 1969 [1927]. 


\section{Références}

BETTELHEIM, Bruno (1982). "Reflections : Freud and the Soul ». The New Yorker, March $1^{\text {tt. }}$.

FREUD, Sigmund (1969). " Le fétichisme ". Dans La vie sexuelle. Paris, Presses Universitaires de France.

GUBRICH-SIMITIS, Ilse (1997). Freud : Retour atxx manuscrits. Paris, Presses Universitaires de France.

LA FONTAINE, Jean de (1991). Fables. Paris, Gallimard (Pléiade).

ROSE, Louis (1988). * Freud and Fetishism : Previously Unpublished Minutes of the Vienna Psychoanalytic Society *. Psychoanalytic Quarterly. LVIl, pp. 147-166.

STEINER, George (1992). After Babel : Aspects of Language and Translation. Oxford, Oxford University Press.

WIENER PSYCHANALYTISCHE VEREINIGUNG (1976-1983) [collectif]. Les premiers psychanalystes : minutes de la Société psychanalytique de Vienne, 4 vol., Paris, Gallimard.

RÉSUMÉ : Interprétation et fétiches : entre traduction et psychanalyse - Si, comme le démontre George Steiner, la traduction n'est finalement qu'un mode d'interprétation, cela soulève immanquablement la question des critères pertinents et éventuellement applicables à l'évaluation de traductions. Pourtant, ce que l'on constate le mieux avec Steiner Iorsqu'il évalue des traductions, c'est toute la complexité des relations entre traducteurs-interprètes et les textes qu'ils traduisent - à la fois au sens qu'elles/ils leur attribuent comme à leurs modes de le signifier. De même, l'évaluation, les jugements que l'on porte sur les traductions en disent long sur celle/celui qui évalue, sur l'importance qu'elle/il attache au sens, voire à la possibilité même de connaître ou de comprendre le monde où nous évoluons.

Tels qu'il les décrit, les premiers cas de fétichisme chez Freud opèrent de façon analogue : ils s'avèrent n'être qu'une série de tentatives 
d'adaptation à la réalité. Les fétiches servent à la fois à commémorer et à nier une réalité perçue mais inacceptable. Ce genre de paradoxe et les simulacres qui servent à déguiser cette réalité ne sont finalement que le tribut qu'il faut payer en échange de ce sentiment rassurant que le monde est disponible à notre entendement, que les moyens très limités dont nous disposons pour le comprendre sont adéquats. Sans critères absolus pour les évaluer, les traductions fonctionnent comme des fétiches : elles prouvent - mais provisoirement - que nous sommes capables de comprendre et d'interpréter le monde, alors que, par leur imperfection, elles réaffirment le fait que telle compréhension n'est finalement que fragmentaire.

\begin{abstract}
Interpretation and Fetishes: Between Translation and Psychoanalysis - If, as George Steiner demonstrates, translation is a form of interpretation, evaluating translations raises the question of knowledge, that of the implicit apprehension of a standard against which any given translation is to be measured. Yet, as Steiner also shows, what translations ultimately reveal most clearly is the intricate relationship the translators as interpreters entertain toward texts, toward their putative meaning and toward their various modes of signifying it. In much the same way, the evaluation of translations reveals the evaluator's own preoccupation with meaning, with the very possibility of knowing or understanding the world.

Freud's earliest cases of fetishism reveal it as a form of adaptation, as an evolving series of attempts to adapt to an unacceptable perception of reality by at once denying and commemorating that perception. Such contradictions and the simulacra which serve to disguise reality are, in the end, the wages paid in exchange for the reassuring sense that the world is knowable, that it is accessible through the very limited means of understanding we have at our disposal. In the absence of any ultimate standard, translations serve the same purposes as fetishes : they at once demonstrate our ability to interpret and understand although, by their inevitable perfectibility, they reiterate the fact that such understanding can only be partial.
\end{abstract}

Gabriel Louis Moyal : Département d'études françaises, McMaster University, 1280 Main Street West, Hamilton (Ontario) L8S 4M2.

Courriel : moyalg@mcmail.cis.mcmaster.ca 